\title{
Naked-Eye Detection of Hepatitis B Surface Antigen Using Gold Nanoparticles Aggregation and Catalase-Functionalized Polystyrene Nanospheres
}

\author{
Rubing Chen, Yongqin Hu, Meizhu Chen, Jia An, Ying Lyu, Yufei Liu,* and Dongling Li* \\ Cite This: ACS Omega 2021, 6, 9828-9833 \\ Read Online
}

ACCESS | Llll Metrics \& More | 国 Article Recommendations | sl Supporting Information

ABSTRACT: Developing rapid, efficient, highly sensitive, simple, stable, and low-cost virus marker detection products that are appropriate for basic facilities is of great importance in the early diagnosis and treatment of viruses. Naked-eye detection methods are especially important when medical testing facilities are limited. Polystyrene nanospheres (PSs) with catalytic and specific recognition functions were successfully developed by simultaneously modifying catalase and goat anti-hepatitis B surface antibodies on nanospheres. The modified PSs contributed significantly to the amplification of the signal. Via the specific antigen-antibody reaction, the bifunctional nanospheres could be captured on microplate and then catalyzed the decomposition of

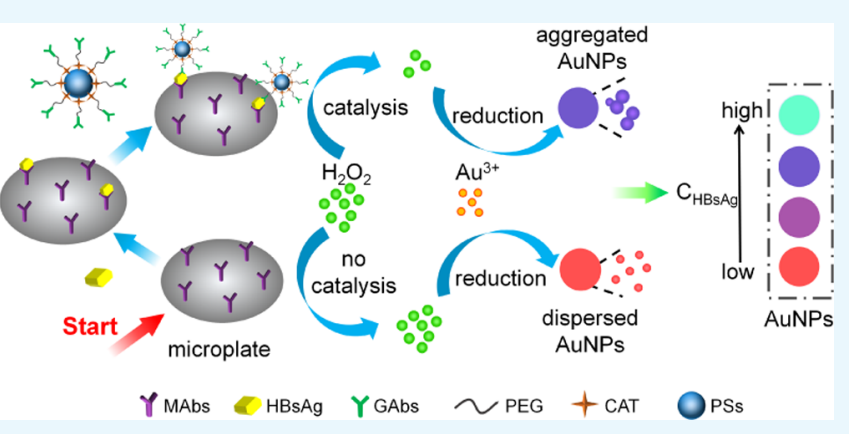
hydrogen peroxide to reduce chloroauric acid and synthesize gold nanoparticles (AuNPs). Due to the surface plasmon resonance of AuNPs, the solution color change could be observed with the naked eye and the limit of detection (LOD) was $0.1 \mathrm{ng} / \mathrm{mL}$. Furthermore, the LOD observed with instrumentation was $0.01 \mathrm{ng} / \mathrm{mL}$, which meant that a rapid, efficient, and highly sensitive method for the detection of hepatitis B surface antigens was successfully developed, and neither complex sample pretreatment nor expensive equipment was needed.

\section{INTRODUCTION}

Viral hepatitis is one of the most serious public health problems. For example, infection with hepatitis B virus (HBV) will lead to hepatitis $B$, which is endemic, widespread, and severely harmful. It can manifest in a variety of clinical types, such as chronic hepatitis, hepatocellular carcinoma, acute hepatitis, and cirrhosis, ${ }^{1,2}$ leading to tens of thousands of deaths each year. At present, there is no effective treatment method for $\mathrm{HBV}$ at home or abroad, and patients can only be treated with nucleoside analogues or interferons to inhibit virus replication and worsening of the liver disease; nevertheless, for most patients, HBV cannot be eliminated. Therefore, early diagnosis of HBV is essential for the effective prevention and treatment of the disease.

The existing methods for detecting HBV include enzymelinked immunosorbent assay (ELISA), ${ }^{3}$ radioimmunoassay (RIA), ${ }^{4}$ chemiluminescence immunoassay (CIA), ${ }^{5}$ and electrochemical immunoassay (EIA). ${ }^{6}$ Using commercially available ELISA to detect viruses is common because of the specific reaction of the antigen and the antibody and enzyme catalysis, but the shortcomings are also apparent; these include low sensitivity and easily missed inspections for low-level people. The RIA method is reliable and accurate, but its use suffers from problems related to health, waste disposal, and the need for expensive equipment. CIA cannot target a single compound but will react to a series of compounds, so its selectivity is poor. Additionally, the selectivity of EIA is usually poor. Therefore, the development of simple, sensitive, and rapid early clinical diagnosis and detection methods for $\mathrm{HBV}$ is essential for human health care.

With the rise and development of nanotechnology, the unique physicochemical properties of nanomaterials have aided in the development of new methods for the sensitive detection of biological analytes, and various nanoparticles, including quantum dots, ${ }^{8}$ nano-porphyrins, ${ }^{9}$ and metal nanoparticles, ${ }^{10}$ have been used in bioanalytical determinations. Colorimetric analysis methods based on the surface plasmon resonance (SPR) of gold nanoparticles (AuNPs), which do not require advanced instrumentation, have successfully attracted attention. Because AuNPs offer the advantages of good biocompatibility, unique optical and electronic properties, and relatively easy manufacturing, they are frequently used as carriers in various biomedical applications. ${ }^{11} \mathrm{~A}$ range of biomacromole-

Received: January 28, 2021

Accepted: March 18, 2021

Published: March 30, 2021 
cules, such as antibodies, oligonucleotides, and aptamers, ${ }^{12}$ can functionalize AuNPs. Biomolecular interactions in biological processes can control their dispersion and aggregation. By monitoring the apparent color change caused by AuNPs, the detection of many kinds of (biological) molecules becomes easy, ${ }^{12-19}$ and this provides an excellent platform for the colorimetric biosensor development. For example, Xiong et al. detected the Enterovirus 71 by the SPR of AuNPs with a limit of detection (LOD) equal to $0.65 \mathrm{ng} / \mathrm{mL}$, which is much lower than the commercial ELISA detection $(4.51 \mathrm{ng} / \mathrm{mL}){ }^{20}$

In this work, we advance a colorimetric detection scheme based on a specific antibody-antigen interaction, catalasemediated growth, and aggregation of AuNPs. The method can detect hepatitis $\mathrm{B}$ surface antigen ( $\mathrm{HBsAg}$ ) directly and in a simple manner. As shown in Scheme 1, the capture antibody

Scheme 1. Schematic Diagram of Naked-Eye Detection of HBsAg Using AuNPs Aggregation and Catalase-

Functionalized PSs

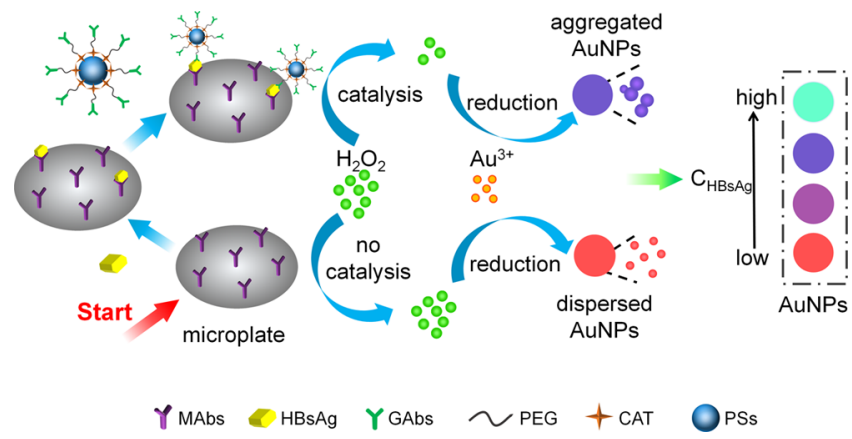

(mouse anti-HBsAg, MAbs) immobilized on a microplate specifically recognized and effectively captured HBsAg and then the HBsAg also combined with polystyrene nanospheres (PSs) that were modified with goat anti-HBsAg (GAbs) and catalase (CAT). The whole system formed an immune sandwich structure complex. More importantly, it also amplified the detection signal. Then, hydrogen peroxide $\left(\mathrm{H}_{2} \mathrm{O}_{2}\right)$ decomposition was catalyzed by CAT on the complex and the remaining $\mathrm{H}_{2} \mathrm{O}_{2}$ reduced the $\mathrm{Au}^{3+}$ and further affected the morphology of the synthetic AuNPs, which resulted in a significant transformation of the solution color (from red to purple and further to blue). The corresponding absorbance change was proportional to the concentration of HBsAg. Therefore, the method achieves a highly sensitive, fast, efficient, and highly specific visual detection of HBsAg. Moreover, it does not require complex sample preparation, precision instruments, or specialized technical personnel; thus, it has broad prospects in clinical diagnosis, especially in resource-needy areas.

\section{RESULTS AND DISCUSSION}

2.1. Determination of the Feasibility of the Plan. Scheme 1 shows the entire process for the visual detection of HBsAg. Only HBsAg was present, and the GAbs on the bifunctional nanospheres could bind to the captured $\mathrm{HBsAg}$ on the microplate. Subsequently, the CAT modified on the bifunctional nanospheres catalyzed the decomposition of $\mathrm{H}_{2} \mathrm{O}_{2}$; this, in turn, affected the morphology of the AuNPs synthesized with chloroauric acid, leading to the visually recognized change in the color of the solution. The higher the

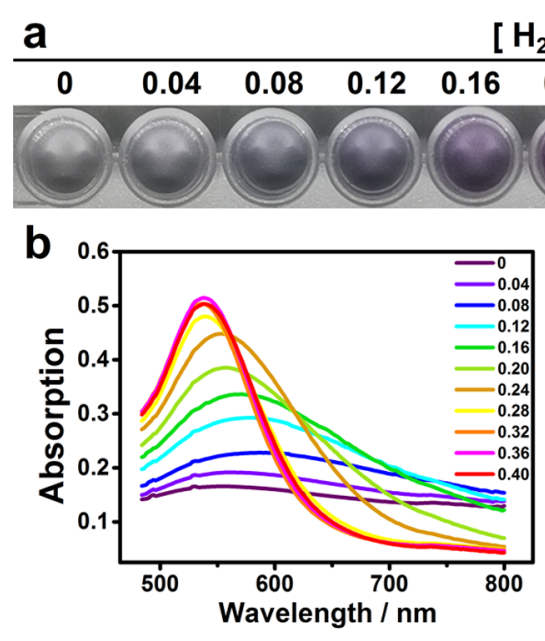

$\left[\mathrm{H}_{2} \mathrm{O}_{2}\right] / \mathrm{mM}$

$\begin{array}{llllll}0.20 & 0.24 & 0.28 & 0.32 & 0.36 & 0.40\end{array}$

d

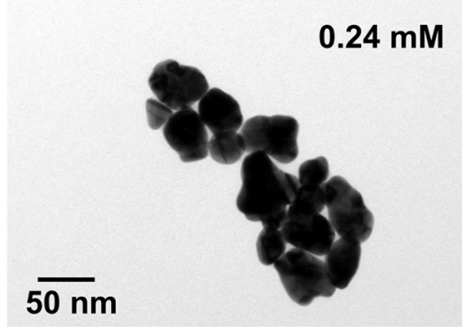

C

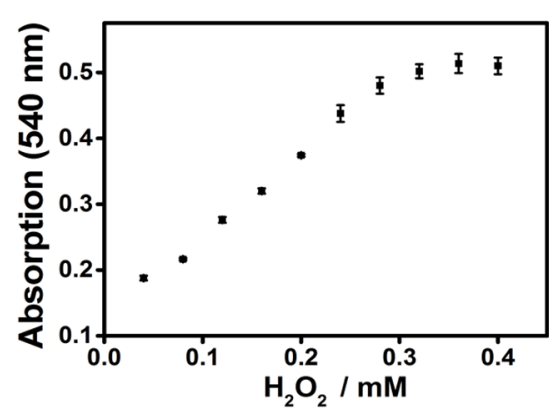

e

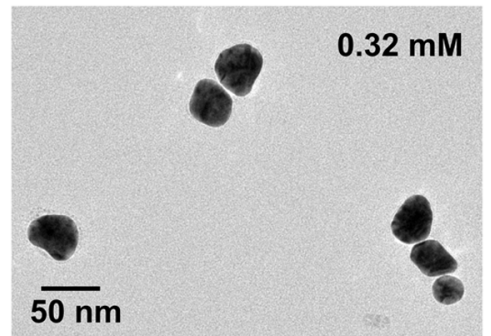

Figure 1. Relationship between the concentration of $\mathrm{H}_{2} \mathrm{O}_{2}$ and the morphology of AuNPs was built. A series of different concentrations of $\mathrm{H}_{2} \mathrm{O}_{2}$ were reacted with $0.34 \mathrm{mM}$ chloroauric acid solution. (a) Color of the solution containing synthesized AuNPs after 30 min of reaction with different concentrations of $\mathrm{H}_{2} \mathrm{O}_{2}$. (b) UV-vis absorption characteristics of the abovementioned different AuNP solutions. When the $\mathrm{H}_{2} \mathrm{O}_{2}$ concentration was less than $0.28 \mathrm{mM}$, the AuNP local SPR peak red-shifted. (c) Absorbance of different AuNPs at $540 \mathrm{~nm}$ varied with the concentration of $\mathrm{H}_{2} \mathrm{O}_{2}$. (d,e) TEM images of AuNPs synthesized when the $\mathrm{H}_{2} \mathrm{O}_{2}$ concentrations were (d) 0.24 and (e) $0.32 \mathrm{mM}$. 

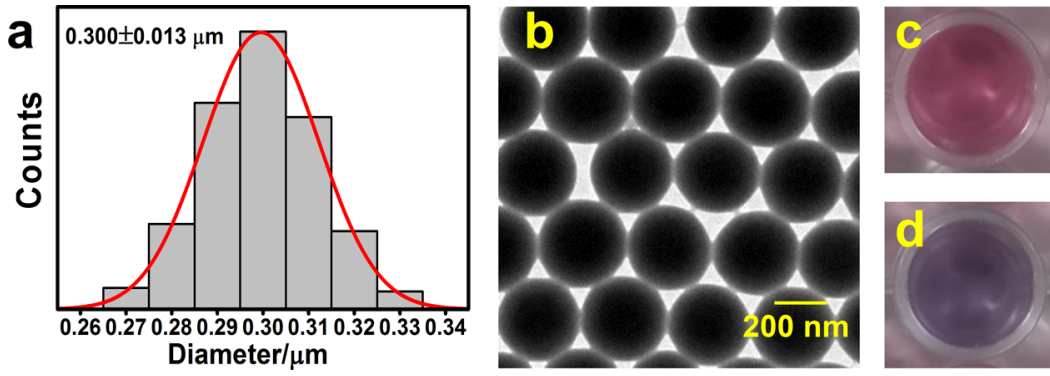

Figure 2. Characterization of PSs and verification of the feasibility of dual-functional PSs. (a) Corresponding size distribution of PSs. (b) TEM images of the PSs. In the presence of HBsAg, (c) when bifunctional nanospheres were not added, the color of the final solution was red, and (d) when bifunctional nanospheres were added, the color of the final solution was blue.

HBsAg concentration was, the greater the color change. Finally, fast, efficient, highly sensitive, and highly specific visual detection of HBsAg was achieved. To ensure the feasibility of the experimental design, it was crucial to prove that the $\mathrm{H}_{2} \mathrm{O}_{2}$ concentration could adjust the morphology and optical properties of the AuNPs synthesized with chloroauric acid.

When a series of different concentrations of $\mathrm{H}_{2} \mathrm{O}_{2}$ were reacted with $0.34 \mathrm{mM}$ chloroauric acid solution, we observed a conspicuous transformation of the solution color from blue to purple to red (Figure 1a), which meant that the concentration of $\mathrm{H}_{2} \mathrm{O}_{2}$ did regulate the morphology of the synthesized AuNPs. This is because, when $\mathrm{H}_{2} \mathrm{O}_{2}$ reduces chloroauric acid, small AuNP seeds are first generated, and then, the generated seeds continue to grow. If the concentration of $\mathrm{H}_{2} \mathrm{O}_{2}$ is low, the nucleation rate for AuNPs will be slower than the growth rate, so it is easy to generate AuNPs with large particle sizes. If the concentration of $\mathrm{H}_{2} \mathrm{O}_{2}$ is high, the nucleation rate of AuNPs will be faster than the growth rate, and it is easy to generate many scattered AuNPs with small particle sizes. As shown in Figure $1 b, c$, when the concentration of $\mathrm{H}_{2} \mathrm{O}_{2}$ was less than $0.28 \mathrm{mM}$, the local SPR spectrum of the AuNPs broadened and redshifted to longer wavelengths. Additionally, transmission electron microscopy (TEM) imaging also confirmed that the final morphology of the synthesized AuNPs was strictly dependent on the concentration of $\mathrm{H}_{2} \mathrm{O}_{2}$ (Figures 1d,e and S1). In short, more viral antigens meant more enzymes were connected, less $\mathrm{H}_{2} \mathrm{O}_{2}$ remained after catalytic decomposition, the synthesized AuNPs were more agglomerated, and the solution color was blue, that is, the experimental scheme was feasible.

2.2. Optimization of Bifunctional Nanospheres. Based on the fact that the final morphology of synthesized AuNPs strictly depended on the concentration of $\mathrm{H}_{2} \mathrm{O}_{2}$, we designed an enzyme-catalyzed reaction in which CAT was used to decompose $\mathrm{H}_{2} \mathrm{O}_{2}$ and affect the morphology of the synthesized AuNPs. Additionally, to achieve rapid detection, we used PSs (approximately $300 \mathrm{~nm}$ in size) made in the laboratory as the carrier (Figure 2a,b). If GAbs, CAT, and PSs are reacted together, competition will occur between GAbs and CAT; GAbs is more competitive than CAT, which will affect the modification process. Therefore, we adopted a layer-by-layer modification method. Considering the issue of competitiveness, we first used CAT to occupy all the modification sites on the PSs and then used poly(ethylene glycol) (PEG) as the connecting arm between CAT and GAbs to increase the distance between them; this would reduce the steric hindrance and enhance the modification effect so that dual-function nanospheres (PSs@CAT@PEG@GAbs) with strong catalytic and specific recognition functions could be obtained. When an antibody molecule on the bifunctional PSs binds to an antigen molecule, all CAT on the complex will participate in the $\mathrm{H}_{2} \mathrm{O}_{2}$ catalytic reaction; therefore, the detection signal would be amplified and the sensitivity could also be improved. As shown in Figure 2c,d, in the presence of HBsAg, the addition of dualfunctional nanospheres significantly affected the color of the final solution, which meant that the dual-functional nanospheres achieved the goal of detecting HBsAg. To ensure high detection sensitivity, the dual-function nanospheres needed to be optimized.

With $1 \mathrm{mg}$ of PSs, the addition of different amounts of CAT for coupling resulted in different enzyme activities for the PSs@CAT obtained. As shown in Figure S2a, when 0.7 mg of CAT was used, the absorbance was the lowest of those observed, indicating that the color of the solution was the lightest and the concentration of $\mathrm{H}_{2} \mathrm{O}_{2}$ remaining after catalysis was the lowest, that is, the highest level of CAT was modified on the PSs and the best enzyme activity for PSs@ CAT was observed. Similarly, as shown in Figure S2b,c, when 1 $\mathrm{mg}$ of PSs, $0.7 \mathrm{mg}$ of CAT, $0.8 \mathrm{mg}$ of PEG, and $30 \mu \mathrm{g}$ of GAbs were fixed, the bifunctional nanospheres in the microplate could catalyze the most $\mathrm{H}_{2} \mathrm{O}_{2}$ and the concentration of $\mathrm{H}_{2} \mathrm{O}_{2}$ remaining was the lowest, so the solution exhibited the lightest color and the lowest absorbance. This meant that the coupling process of the dual-functional PSs had reached the optimal level, and subsequent testing could be carried out.

2.3. Detection of HBsAg. After obtaining the optimal dual-functional nanospheres, we sought to optimize each step of the detection to further improve the detection sensitivity. The specific optimization conditions are shown in Figure S3. Because the absorbances of the solutions in the experimental group at $540 \mathrm{~nm}$ were lower than that in the control group, it was easy to use these differences $\left(\Delta A_{540}\right)$ to distinguish successful and unsuccessful experiments. The larger $\Delta A_{540}$ was, the greater the color difference of the solution, the lighter the color of the solution in the experimental group with $\mathrm{HBsAg}$ added, which meant the detection sensitivity of $\mathrm{HBsAg}$ was higher under this experimental conditions. That is, the larger $\Delta A_{540}$ signified better conditions. The remaining detection conditions were optimized as follows: the concentration of the capture antibody (MAbs) coated on the microplate was $2 \mu \mathrm{g} /$ $\mathrm{mL}$, the reaction time for the specific binding of $\mathrm{HBsAg}$ and the MAbs was $75 \mathrm{~min}$, the reaction time for the HBsAg that had been captured on the microplate specifically bound to the GAbs on the bifunctional nanosphere was $45 \mathrm{~min}$, the reaction time to catalyze the decomposition of $\mathrm{H}_{2} \mathrm{O}_{2}$ by CAT on the dual-functional nanospheres binding to the microplate was 30 min, and, after decomposition, the reaction time for the remaining $\mathrm{H}_{2} \mathrm{O}_{2}$ reducing $\mathrm{Au}^{3+}$ to produce AuNPs was $10 \mathrm{~min}$. 


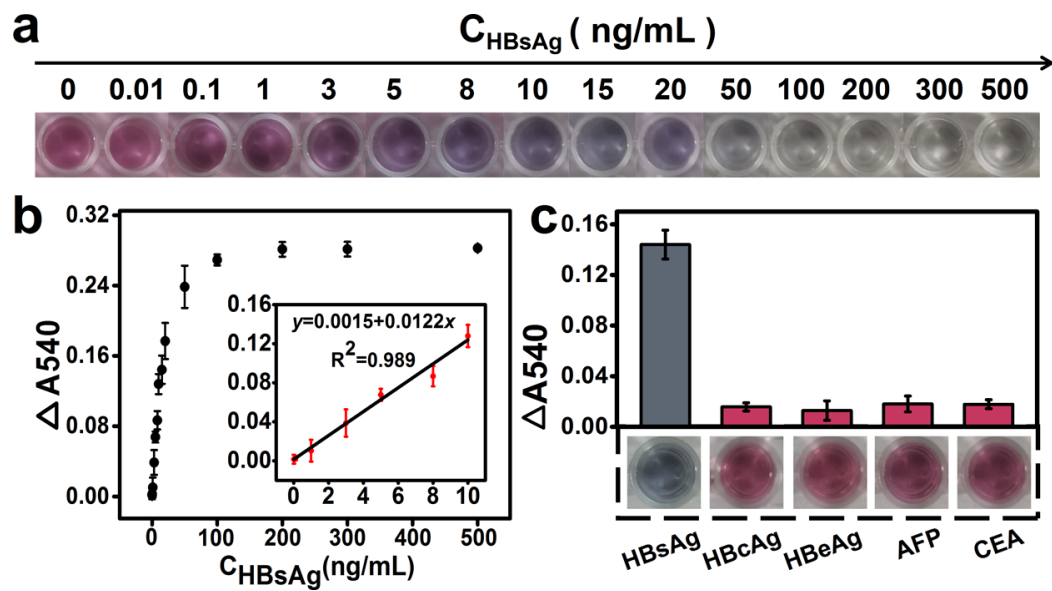

Figure 3. Proposed colorimetric method was used to detect HBsAg. With increasing HBsAg concentration, (a) change in the solution color and (b) change in absorbance at $540 \mathrm{~nm}\left(\Delta A_{540}\right)$, inset: at low HBsAg concentrations, the concentration was linearly related to absorbance. Other common markers, such as hepatitis B core antigen ( $\mathrm{HBcAg}$ ), hepatitis B e antigen ( $\mathrm{HBeAg}$ ), carcinoembryonic antigen (CEA), and $\alpha$-fetoprotein (AFP) antigen, were used as negative samples, and $2 \mathrm{mg} / \mathrm{mL}$ PBS-BSA solution was used as a control to verify the specificity of the method. (c) Color of the solution and the absorbance of the solution at $540 \mathrm{~nm}$ after addition of the samples.

Under these optimal conditions, different concentrations of HBsAg were tested. As shown in Figure $3 a$, when the HBsAg level increased, the number of combined bifunctional PSs increased, and the amount of $\mathrm{H}_{2} \mathrm{O}_{2}$ decomposed also increased, which greatly affected the morphology of the synthesized AuNPs. Additionally, the hue of the solution also transformed from red into purple, then into blue, and finally became colorless. To accurately measure the concentration of $\mathrm{HBsAg}$, the decrease in the absorbance of the experimental group solution measured at $540 \mathrm{~nm}$ relative to that of the blank control $\left(\Delta A_{540}\right)$ is plotted in Figure $3 \mathrm{~b}$. As shown in Figure $3 \mathrm{~b}$, when the concentration of HBsAg increases, $\Delta A_{540}$ also increases, indicating that a linear relationship exists in the range 0.01 and $10 \mathrm{ng} / \mathrm{mL}$, and the linear correlation coefficient $\left(R^{2}\right)$ is 0.989 (inset of Figure $3 \mathrm{~b}$ ). The naked-eye LOD was 0.1 $\mathrm{ng} / \mathrm{mL}$ and the instrumental LOD was $0.01 \mathrm{ng} / \mathrm{mL}$, indicating that the immunocolorimetric assay in this paper could detect HBsAg with high sensitivity.

Furthermore, we compared the method proposed in this article with other traditional methods, such as ELISA, polymerase chain reaction (PCR), EIA, and so forth. As shown in Table 1, comprehensive sensitivity and instrument requirements indicated the superiority of the proposed method in naked-eye detection, which is suitable for resource-poor areas.

\section{Table 1. Comparison of Different Detection Methods}

\begin{tabular}{lllc} 
method & detection object & \multicolumn{1}{c}{ LOD } & references \\
ELISA & HBsAg & $0.5 \mathrm{IU} / \mathrm{mL}$ & 21 \\
CIA & HBsAg & $14 \mathrm{pg} / \mathrm{mL}$ & 22 \\
lateral flow & HBsAg & $75 \mathrm{pg} / \mathrm{mL}$ & 23 \\
PCR & HBV DNA & $2000 \mathrm{IU} / \mathrm{mL}$ & 24 \\
EIA & HBsAg & $0.343 \mathrm{pg} / \mathrm{mL}$ & 17
\end{tabular}

2.4. Specificity Verification. To evaluate the specificity of the method for the detection of $\mathrm{HBsAg}$, other common markers, such as $\mathrm{HBcAg}, \mathrm{HBeAg}, \mathrm{CEA}$, and AFP antigen, were used as negative samples, and $2 \mathrm{mg} / \mathrm{mL}$ PBS-BSA solution was used as a control. As shown in Figure 3c, the blue solution color caused by the aggregation of AuNPs could barely be observed in the presence of HBsAg. On the contrary, in the experiments with negative samples, because the markers could not combine with immune complexes, they were easily washed away when the microplate was washed, so the $\mathrm{H}_{2} \mathrm{O}_{2}$ added subsequently would not be catalyzed, and red scattered AuNPs were ultimately observed. Thus, we know that the color transformation of the solution was the result of the biospecific interaction between HBsAg and the corresponding specific antibody, indicating that our method has high specificity. Additionally, a microplate reader was used to verify the naked eye test results. Based on the decrease in absorbance at $540 \mathrm{~nm}$ $\left(\Delta A_{540}\right)$, the negative and positive samples could be easily distinguished. These results indicate that this method has high selectivity for the identification of HBsAg.

2.5. Detection of HBsAg in Spiked Serum Samples. To further evaluate the applicability of the immunoassay for detecting HBsAg in the human serum, different concentrations of HBsAg were added to the serum, which was diluted 40 times, and then, the detection was carried out under the optimal conditions. As shown in Table 2, recoveries of $\mathrm{HBsAg}$

Table 2. Detecting HBsAg in Spiked Serum Samples by the Proposed Immunoassay

\begin{tabular}{ccccc} 
sample & spiked $(\mathrm{ng} / \mathrm{mL})$ & detected $(\mathrm{ng} / \mathrm{mL})$ & recovery $(\%)$ & RSD (\%) \\
1 & 0.5 & 0.467 & 93.44 & 0.04 \\
2 & 1 & 1.0467 & 104.67 & 0.57 \\
3 & 4 & 4.161 & 104.04 & 3.61 \\
4 & 8 & 7.863 & 98.29 & 2.95 \\
5 & 10 & 10.284 & 102.84 & 2.98 \\
\hline
\end{tabular}

at different concentrations $(0.5-10 \mathrm{ng} / \mathrm{mL})$ in the serum samples are between 93.44 and $104.67 \%$, the average recovery is $100.66 \%$, and the relative standard deviation (RSD) is between 0.04 and $3.61 \%$, indicating that the colorimetric immunoassay is suitable for detecting HBsAg in serum samples and have great prospects for the application in actual clinical practice. 


\section{CONCLUSIONS}

In conclusion, the immunoassay method involving the CATmediated reduction of chloroauric acid to synthesize AuNPs has been successfully used to rapidly and visually detect HBsAg. It provides a simple way of reading results with the naked eye, using the amplification effect of the PSs and the enzyme-catalyzed reaction; this allows the detection of $\mathrm{HBsAg}$ with high efficiency, high sensitivity (instrumental LOD $=0.01$ $\mathrm{ng} / \mathrm{mL}$ ), and high specificity, allowing the use of the method in resource-poor rural units. At the same time, the recovery levels in serum samples with our proposed method are between 93.44 and $104.67 \%$ and the RSD is between 0.04 and 3.61\%, further indicating that the novel method has the great advantages of high specificity and low interference. The visual detection of HBsAg using immune amplification to catalyze the synthesis of AuNPs offers great potential for sensitive, reliable, convenient, and low-cost medical and point-of-care diagnosis.

\section{MATERIALS AND METHODS}

4.1. Materials. Microplates were purchased from JET. The ultrafiltration tube $(50 \mathrm{k})$ was purchased from Bioground (Chongqing). $\mathrm{HBsAg}, \mathrm{HBcAg}, \mathrm{HBeAg}$, enzyme-labeled antibody diluent, GAbs, and MAbs were purchased from Bai Aotong (Luoyang). 2-(4-Morpholino)ethanesulfonic acid (MES), catalase, and $N$-(3-dimethylaminopropyl)- $N^{\prime}$-ethylcarbodiimide hydrochloride (EDC) were purchased from Sigma. $\mathrm{NH}_{2}-\mathrm{PEG}-\mathrm{COOH}$ was purchased from Peng Sheng Biological (Shanghai). The PSs-COOH $(300 \mathrm{~nm})$ was made in the laboratory. Chloroauric acid $\left(\mathrm{HAuCl}_{4}\right)$ was purchased from Macklin. BSA was purchased from BioFroxx. The remaining drugs were bought from Chron Chemicals. All the experiments used ultrapure water $(18.2 \mathrm{M} \Omega \mathrm{cm})$.

4.2. Preparation of PSs@CAT@PEG@GAbs. First, the CAT must be purified because the purchased CAT solution is a suspension in which the CAT is not sufficiently dissolved. A part of the CAT stock solution was centrifuged, the supernatant was collected, and the precipitate was dispersed in $0.01 \mathrm{M}$ PBS buffer $(\mathrm{pH}=7.2)$ and centrifuged until the supernatant was colorless and transparent. After combining all the supernatants, an ultrafiltration tube was used to concentrate the samples, and the samples were washed with $0.01 \mathrm{M}$ PBS buffer $(\mathrm{pH}=7.2)$. Eventually, the remaining liquid in the ultrafiltration tube was adjusted to a constant volume and concentration, and the resulting liquid was used for the CAT experiment and stored at $4{ }^{\circ} \mathrm{C}$.

The preparation of carboxylated PSs was described by Xie et al. $^{25}$ The preparation of PSs@CAT@PEG@GAbs was described by $\mathrm{Hou}$ et al., and some improvements were made. $^{26}$ PSs $(1 \mathrm{mg}), 0.7 \mathrm{mg}$ of CAT, and $10 \mathrm{~mL}$ of $0.01 \mathrm{M}$ PBS buffer were added to a round-bottom flask. After stirring at $25{ }^{\circ} \mathrm{C}$ for $1 \mathrm{~h}, 300 \mu \mathrm{L}$ of the EDC solution was added and stirring was continued for $4 \mathrm{~h}$. The mixture was washed once with 1X PBS buffer to obtain the PSs@CAT complex and then stored at $4{ }^{\circ} \mathrm{C}$. The methods for the preparation of PSs@ CAT@PEG and PSs@CAT@PEG@GAbs were the same as that for the PSs@CAT complex.

4.3. Detection of HBsAg. First, $100 \mu \mathrm{L}$ of $2 \mu \mathrm{g} / \mathrm{mL}$ MAbs solution prepared with $\mathrm{CB}$ buffer was added to each well of the microplate and washed with PBST buffer after reacting at 37 ${ }^{\circ} \mathrm{C}$ for $2 \mathrm{~h}$. Then, the plate was blocked with $1 \times$ PBS solution (containing $20 \mathrm{mg} / \mathrm{mL} \mathrm{BSA}$ ) for $1 \mathrm{~h}$ and washed with PBST buffer. It was then stored at $4{ }^{\circ} \mathrm{C}$.
In a microplate coated with MAbs, $100 \mu \mathrm{L}$ of HBsAg solution diluted with $2 \mathrm{mg} / \mathrm{mL}$ BSA solution (containing $0.05 \%$ Tween 20 , prepared with $1 \times$ PBS buffer) was added to different concentrations in the sequence and then washed with PBST buffer after reacting at $37^{\circ} \mathrm{C}$ for $75 \mathrm{~min}$. Then, $20 \mu \mathrm{L}$ of PSs@CAT@PEG@GAbs and80 $\mu \mathrm{L}$ of enzyme-labeled antibody diluent were added. After reacting at $37{ }^{\circ} \mathrm{C}$ for 45 min, the cells were washed with PBST buffer 3 times, $1 \times$ PBS buffer twice, and water once. Subsequently, a certain amount of $\mathrm{H}_{2} \mathrm{O}_{2}$ diluted with $1 \mathrm{mM}$ MES buffer (the $\mathrm{pH}$ of the buffer was adjusted to 6.50 with $\mathrm{NaOH}$ ) was added to each well. After reacting at $37^{\circ} \mathrm{C}$ for $30 \mathrm{~min}$, the chloroauric acid solution was added to react for $30 \mathrm{~min}$ at $37^{\circ} \mathrm{C}$. A microplate reader was used to detect the absorbance of the solution at $540 \mathrm{~nm}$. According to the difference in the absorbance between the control group and the experimental group, we established a linear relationship between the difference in the absorbance and the corresponding HBsAg concentration.

The specific steps of detection were the same as the steps for detecting HBsAg in PBS buffer.

4.4. HBsAg Detection in Human Serum. To verify the feasibility of this method for testing actual samples, HBsAg was added to a normal human serum (from a volunteer) diluted 40-fold. The added HBsAg concentrations were 0.5, 1, 4, 8, and $10 \mathrm{ng} / \mathrm{mL}$.

\section{ASSOCIATED CONTENT}

\section{Supporting Information}

The Supporting Information is available free of charge at https://pubs.acs.org/doi/10.1021/acsomega.1c00507.

TEM images of the AuNPs formed with different concentrations of $\mathrm{H}_{2} \mathrm{O}_{2}$, optimal amounts of CAT, PEG, and GAbs used for $1 \mathrm{mg}$ of PSs, and the optimization of specific detection steps (PDF)

\section{AUTHOR INFORMATION}

\section{Corresponding Authors}

Yufei Liu - Key Laboratory of Optoelectronic Technology \& Systems (Chongqing University), Ministry of Education, Chongqing 400044, China; Center for Intelligent Sensing Technology (CIST), College of Optoelectronic Engineering, Chongqing University, Chongqing 400044, China; Centre for NanoHealth, College of Science, Swansea University, Swansea SA2 8PP, U.K.; 1 orcid.org/0000-0003-2988-8843; Email: Yufei.Liu@cqu.edu.cn

Dongling Li - Key Laboratory of Optoelectronic Technology \& Systems (Chongqing University), Ministry of Education, Chongqing 400044, China; Email: lidongling@cqu.edu.cn

\section{Authors}

Rubing Chen - Key Laboratory of Optoelectronic Technology \& Systems (Chongqing University), Ministry of Education, Chongqing 400044, China

Yongqin $\mathbf{H u}-$ Key Laboratory of Optoelectronic Technology \& Systems (Chongqing University), Ministry of Education, Chongqing 400044, China; Center for Intelligent Sensing Technology (CIST), College of Optoelectronic Engineering, Chongqing University, Chongqing 400044, China

Meizhu Chen - Key Laboratory of Optoelectronic Technology \& Systems (Chongqing University), Ministry of Education, Chongqing 400044, China 
Jia An - Key Laboratory of Optoelectronic Technology \& Systems (Chongqing University), Ministry of Education, Chongqing 400044, China; Center for Intelligent Sensing Technology (CIST), College of Optoelectronic Engineering, Chongqing University, Chongqing 400044, China

Ying Lyu - Key Laboratory of Optoelectronic Technology \& Systems (Chongqing University), Ministry of Education, Chongqing 400044, China

Complete contact information is available at: https://pubs.acs.org/10.1021/acsomega.1c00507

\section{Notes}

The authors declare no competing financial interest.

\section{ACKNOWLEDGMENTS}

The project was supported by the Fundamental Research Funds for the Central Universities of China (grant no. 2019CDQYGD020 and no. 2019CDCGGD304), the National Key Research and Development Program of China (grant no. 2016YFE0125200) and National Natural Science Foundation of China (grant no. 61927818). We thank for the test platform provided by the Analytical and Testing Center of Chongqing University and Beijing Zhongkebaice Technology Service Co., Ltd.

\section{REFERENCES}

(1) Lavanchy, D. Worldwide epidemiology of HBV infection, disease burden, and vaccine prevention. J. Clin. Virol. 2005, 34, S1-S3.

(2) Ocama, P.; Opio, C. K.; Lee, W. M. Hepatitis B virus infection: current status. Am. J. Med. 2005, 118, 1413.

(3) Yamamoto, Y.; Saita, T.; Sogawa, R.; Ogata, K.; Yamamoto, Y.; Kimura, S.; Narisawa, Y.; Kimura, S.; Shin, M. Development of a sandwich enzyme-linked immunosorbent assay for the quantification of ponatinib in serum. Anal. Biochem. 2019, 571, 14-20.

(4) Franco-Martinez, L.; Tvarijonaviciute, A.; Martinez-Subiela, S.; Teles, M.; Tort, L. Chemiluminescent assay as an alternative to radioimmunoassay for the measurement of cortisol in plasma and skin mucus of Oncorhynchus mykiss. Ecol. Indic. 2019, 98, 634-640.

(5) Ehsani, M.; Chaichi, M. J.; Nezammeddin Hosseini, S. Comparison of $\mathrm{CuO}$ nanoparticle and $\mathrm{CuO} / \mathrm{MWCNT}$ nanocomposite for amplification of chemiluminescence immunoassay for detection of the hepatitis B surface antigen in biological samples. Sens. Actuators, B 2017, 247, 319-328.

(6) Mandli, J.; Attar, A.; Ennaji, M. M.; Amine, A. Indirect competitive electrochemical immunosensor for hepatitis A virus antigen detection. J. Electroanal. Chem. 2017, 799, 213-221.

(7) Giljohann, D. A.; Mirkin, C. A. Drivers of biodiagnostic development. Nature 2009, 462, 461-464.

(8) Yang, X.; Xu, J.; Luo, N.; Tang, F.; Zhang, M.; Zhao, B. N,Cl codoped fluorescent carbon dots as nanoprobe for detection of tartrazine in beverages. Food Chem. 2020, 310, 125832.

(9) Paolesse, R.; Nardis, S.; Monti, D.; Stefanelli, M.; Di Natale, C. Porphyrinoids for Chemical Sensor Applications. Chem. Rev. 2017, 117, 2517-2583.

(10) Yu, L.; Li, N. Noble Metal Nanoparticles-Based Colorimetric Biosensor for Visual Quantification: A Mini Review. Chemosensors 2019, 7, 53 .

(11) Li, Y.; Schluesener, H. J.; Xu, S. Gold nanoparticle-based biosensors. Gold Bull. 2010, 43, 29-41.

(12) Saha, K.; Agasti, S. S.; Kim, C.; Li, X.; Rotello, V. M. Gold Nanoparticles in Chemical and Biological Sensing. Chem. Rev. 2012, 112, 2739-2779.

(13) Cordeiro, M.; Carlos, F. F.; Pedrosa, P.; Lopez, A.; Baptista, P. V. Gold Nanoparticles for Diagnostics: Advances towards Points of Care. Diagnostics 2016, 6, 43.
(14) Hu, J.; Wang, L.; Li, F.; Han, Y. L.; Lin, M.; Lu, T. J.; Xu, F. Oligonucleotide-linked gold nanoparticle aggregates for enhanced sensitivity in lateral flow assays. Lab Chip 2013, 13, 4352-4357.

(15) Kamińska, A.; Witkowska, E.; Winkler, K.; Dziecielewski, I.; Weyher, J. L.; Waluk, J. Detection of Hepatitis B virus antigen from human blood: SERS immunoassay in a microfluidic system. Biosens. Bioelectron. 2015, 66, 461-467.

(16) Kim, D. S.; Kim, Y. T.; Hong, S. B.; Kim, J.; Heo, N. S.; Lee, M.-K.; Lee, S. J.; Kim, B. I.; Kim, I. S.; Huh, Y. S.; Choi, B. G. Development of Lateral Flow Assay Based on Size-Controlled Gold Nanoparticles for Detection of Hepatitis B Surface Antigen. Sensors 2016, 16, 2154

(17) Shourian, M.; Ghourchian, H.; Boutorabi, M. Ultra-sensitive immunosensor for detection of hepatitis B surface antigen using multi-functionalized gold nanoparticles. Anal. Chim. Acta 2015, 895, $1-11$.

(18) Veigas, B.; Pedrosa, P.; Carlos, F. F.; Mancio-Silva, L.; Grosso, A. R.; Fortunato, E.; Mota, M. M.; Baptista, P. V. One nanoprobe, two pathogens: gold nanoprobes multiplexing for point-of-care. $J$. Nanobiotechnol. 2015, 13, 48.

(19) Wu, Y.; Guo, W.; Peng, W.; Zhao, Q.; Piao, J.; Zhang, B.; Wu, X.; Wang, H.; Gong, X.; Chang, J. Enhanced Fluorescence ELISA Based on HAT Triggering Fluorescence "Turn-on" with EnzymeAntibody Dual Labeled AuNP Probes for Ultrasensitive Detection of AFP and HBsAg. ACS Appl. Mater. Interfaces 2017, 9, 9369-9377.

(20) Xiong, L.-H.; He, X.; Xia, J.; Ma, H.; Yang, F.; Zhang, Q.; Huang, D.; Chen, L.; Wu, C.; Zhang, X.; Zhao, Z.; Wan, C.; Zhang, R.; Cheng, J. Highly Sensitive Naked-Eye Assay for Enterovirus 71 Detection Based on Catalytic Nanoparticle Aggregation and Immunomagnetic Amplification. ACS Appl. Mater. Interfaces 2017, 9, 14691-14699.

(21) van den Berg, L. M.; Ribeiro, C. M. S.; Zijlstra-Willems, E. M.; de Witte, L.; Fluitsma, D.; Tigchelaar, W.; Everts, V.; Geijtenbeek, T. B. H. Caveolin-1 mediated uptake via langerin restricts HIV-1 infection in human Langerhans cells. Retrovirology 2014, 11, 123.

(22) Sabouri, S.; Ghourchian, H.; Shourian, M.; Boutorabi, M. A gold nanoparticle-based immunosensor for the chemiluminescence detection of the hepatitis B surface antigen. Anal. Methods 2014, 6, 5059-5066.

(23) Shen, J.; Zhou, Y.; Fu, F.; Xu, H.; Lv, J.; Xiong, Y.; Wang, A. Immunochromatographic assay for quantitative and sensitive detection of hepatitis B virus surface antigen using highly luminescent quantum dot-beads. Talanta 2015, 142, 145-149.

(24) Price, H.; Dunn, D.; Zachary, T.; Vudriko, T.; Chirara, M.; Kityo, C.; Munderi, P.; Spyer, M.; Hakim, J.; Gilks, C.; Kaleebu, P.; Pillay, D.; Gilson, R.; Grp, D. V. Hepatitis B serological markers and plasma DNA concentrations. AIDS 2017, 31, 1109-1117.

(25) Xie, H.-Y.; Zuo, C.; Liu, Y.; Zhang, Z.-L.; Pang, D.-W.; Li, X.L.; Gong, J.-P.; Dickinson, C.; Zhou, W. Cell-Targeting Multifunctional Nanospheres with both Fluorescence and Magnetism. Small 2005, 1, 506-509.

(26) Hou, Y.; Cai, G.; Zheng, L.; Lin, J. A microfluidic signal-off biosensor for rapid and sensitive detection of Salmonella using magnetic separation and enzymatic catalysis. Food Control 2019, 103, 186-193. 